\title{
Ophthalmology research in the UK's National Health Service: the structure and performance of the NIHR's Ophthalmology research portfolio
}

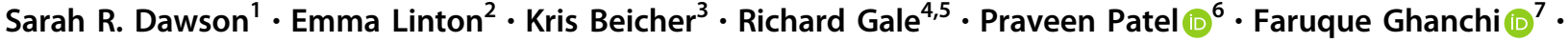 \\ Michael W. Beresford ${ }^{8,9} \cdot$ Vanessa Poustie $^{10} \cdot$ Usha Chakravarthy $^{11}$. \\ Rupert R. A. Bourne ${ }^{12,13} \cdot$ NIHR Ophthalmology Specialty Group
}

Received: 24 August 2018 / Accepted: 30 September 2018 / Published online: 20 November 2018

(c) The Royal College of Ophthalmologists 2018

\begin{abstract}
Purpose To report on the composition and performance of the portfolio of Ophthalmology research studies in the United Kingdom's National Institute for Health Research (NIHR) Clinical Research Network (UK CRN).

Methods Ophthalmology studies open to recruitment between 1 April 2010 and 31 March 2018 were classified by: subspecialty, participant age, gender of Chief Investigator, involvement of genetic investigations, commercial/ non-commercial, interventional/observational design. Frequency distributions for each covariate and temporal variation in recruitment to time and target were analysed.

Results Over 8 years, 137,377 participants were recruited (average of 15,457 participants/year; range: 5485-32,573) with growth by year in proportion of commercial studies and hospital participation in England (76\% in 2017/18). Fourteen percent of studies had a genetic component and most studies (82\%) included only adults. The majority of studies $(41 \%)$ enrolled patients with retinal diseases, followed by glaucoma (17\%), anterior segment and cataract (13\%), and ocular inflammation (6\%). Overall, $68 \%$ of non-commercial studies and $55 \%$ of commercial studies recruited within the anticipated time set by the study and also recruited to or exceeded the target number of participants.

Conclusions High levels of clinical research activity, growth and improved performance have been observed in Ophthalmology in UK over the past 8 years. Some sub-specialties that carry substantial morbidity and a very high burden on NHS services are underrepresented and deserve more patient-centred research. Yet the NIHR and its CRN Ophthalmology National Specialty Group has enabled key steps in achieving the goal of embedding research into every day clinical care.
\end{abstract}

Electronic supplementary material The online version of this article (https://doi.org/10.1038/s41433-018-0251-8) contains supplementary material, which is available to authorized users.

Members of the NIHR Ophthalmology Specialty Group are listed before the references.

These authors share first authorship: Sarah R. Dawson, Emma Linton.

Rupert R. A. Bourne (Senior Author)

rb@rupertbourne.co.uk

1 Wolverhampton Eye Infirmary, The Royal Wolverhampton Hospitals NHS Trust, Wolverhampton, UK

2 Manchester Royal Eye Hospital, Manchester, UK

3 National Institute for Health Research (NIHR CRN), University of Leeds, Leeds, UK

4 Academic Unit of Ophthalmology, York NHS Teaching Hospital, York, UK

5 Department of Health Sciences, University of York, Heslington, UK

\section{Introduction}

The National Institute for Health Research (NIHR) has transformed research in the United Kingdom's National Health Service (NHS) since its establishment in 2006. It has increased the volume of applied health research for the

NIHR Moorfields Biomedical Research Centre, London, UK

Bradford Teaching Hospitals, Bradford, UK

Institute of Translational Medicine, University of Liverpool, Liverpool, UK

9 Department of Paediatric Rheumatology, Alder Hey Children's NHS Foundation Trust, Liverpool, UK

10 Institute of Translational Medicine, University of Liverpool, Liverpool, UK

11 Queen's University of Belfast, Belfast, UK

12 Cambridge University Hospitals, Cambridge, UK

13 Vision \& Eye Research Unit, School of Medicine, Anglia Ruskin University, Cambridge, UK 
benefit of patients and the public, driven faster translation of basic science discoveries into tangible benefits for patients and the economy, and developed and supported the people who conduct and contribute to applied health research. The Health and Social Care Act 2012 places a statutory duty to promote research, and powers to support it, on the Secretary of State and on all levels of the NHS including NHS England, and Clinical Commissioning Groups [1].

The NIHR provides the support and facilities the NHS needs for first-class research by funding a range of infrastructure facilities (Fig. 1). An integral part of this structure is the Clinical Research Network (CRN, received core funding of $£ 302.4$ million in $2017 / 18$ from UK Government's NIHR allocation of $£ 1$ billion) that provides infrastructure and facilitates participation in research.

The NIHR CRN is responsible for creating and maintaining the UK CRN portfolio, a collection of high-quality clinical studies, many of which are randomized controlled trials [2]. Only studies included in this database have access to infrastructure and NHS service support costs offered by the NIHR CRN. NHS service support costs are the costs associated with those tasks which are being undertaken for patient care, but would not be part of standard care, i.e. are not a part of the treatment or regime and end when the research ends. This includes tasks such as extra blood tests, or extra nurse appointments to monitor safety during a trial, but they also cover "ethical safety" such as making sure informed consent is achieved for all participants. The processing of patient records and subsequent mailing activities are considered as NHS service support costs because they make certain that patients are offered the chance to participate only in research opportunities for which they are potentially eligible.

The CRN is divided across the 15 regions of England (termed Local Clinical Research Networks, LCRNs) and supports the set-up and timely delivery of commercial and non-commercial studies in the NHS. Ophthalmologists and optometrists representing each of these regions along with nominated leads for Wales, Scotland, and Northern Ireland (Appendix 1 Supplementary materials) comprise the NIHR CRN Ophthalmology National Specialty Group (https://www.rcophth.ac.uk/professional-resources/resea rch-hub/the-nihr-ophthalmology-clinical-research-network/ the-nihr-ophthalmology-specialty-group/).

The NIHR CRN Ophthalmology National Specialty Group (NSG) oversees, monitors and supports the delivery of Ophthalmology portfolio studies on the UK CRN portfolio. Since the inception of the National Specialty Groups, the Ophthalmology NSG has created a portfolio that has shown steady growth of both commercial and noncommercial studies, and put in place measures to overcome barriers to patient recruitment, and as a consequence high proportions of the portfolio studies have recruited to time and to target (i.e, recruitment of total sample size within the timeframe specified by the chief investigator of the study) as a measure of performance.

In the present report, the NIHR Ophthalmology NSG examines the Ophthalmology portfolio performance in detail to better understand the composition of research undertaken, activity by hospital Trust, sources of funding and the gender balance of the leaders of the projects [3].
Fig. 1 Infrastructure facilities funded by the NIHR (reproduced with permission of NIHR)

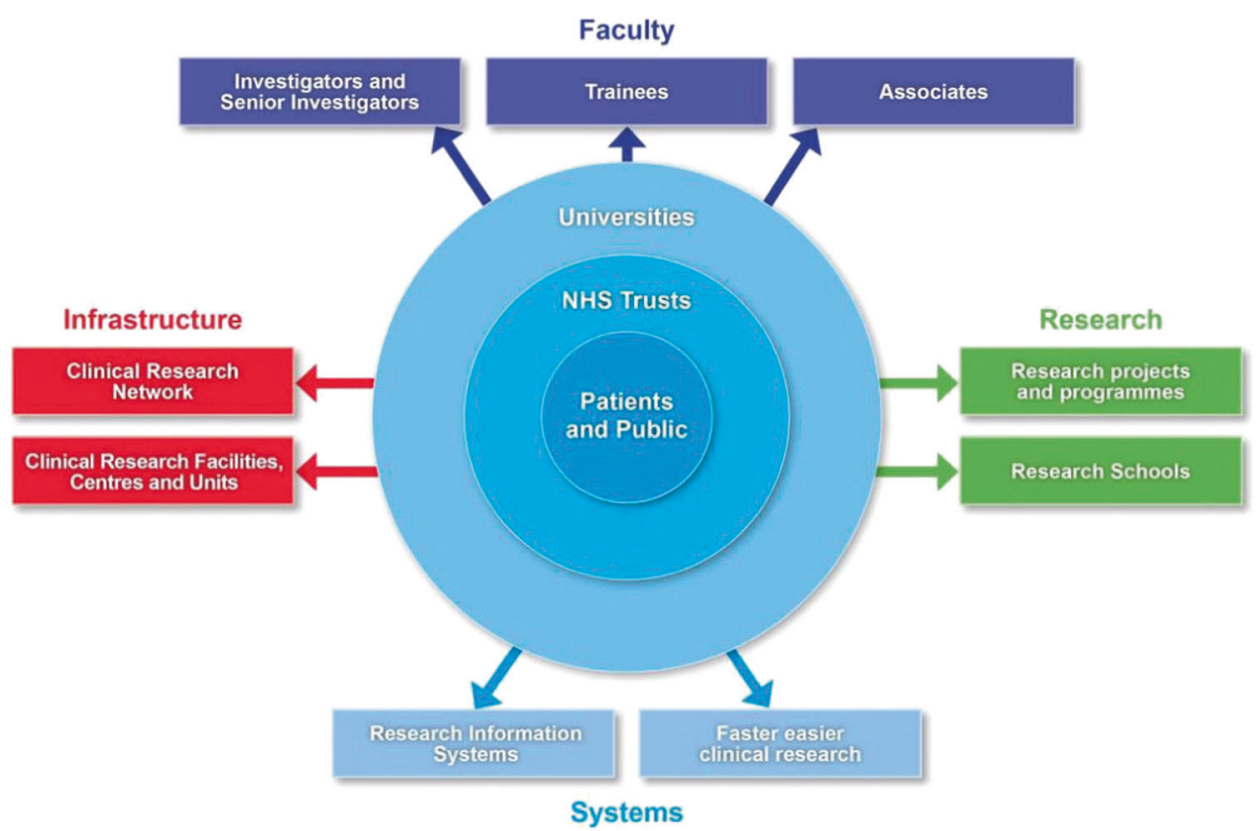


Fig. 2 Numbers of participants recruited into NIHR CRN Ophthalmology portfolio studies in the UK by year, with categorisation into commercial and non-commercial sponsorship, and by design (observational/interventional/ both)

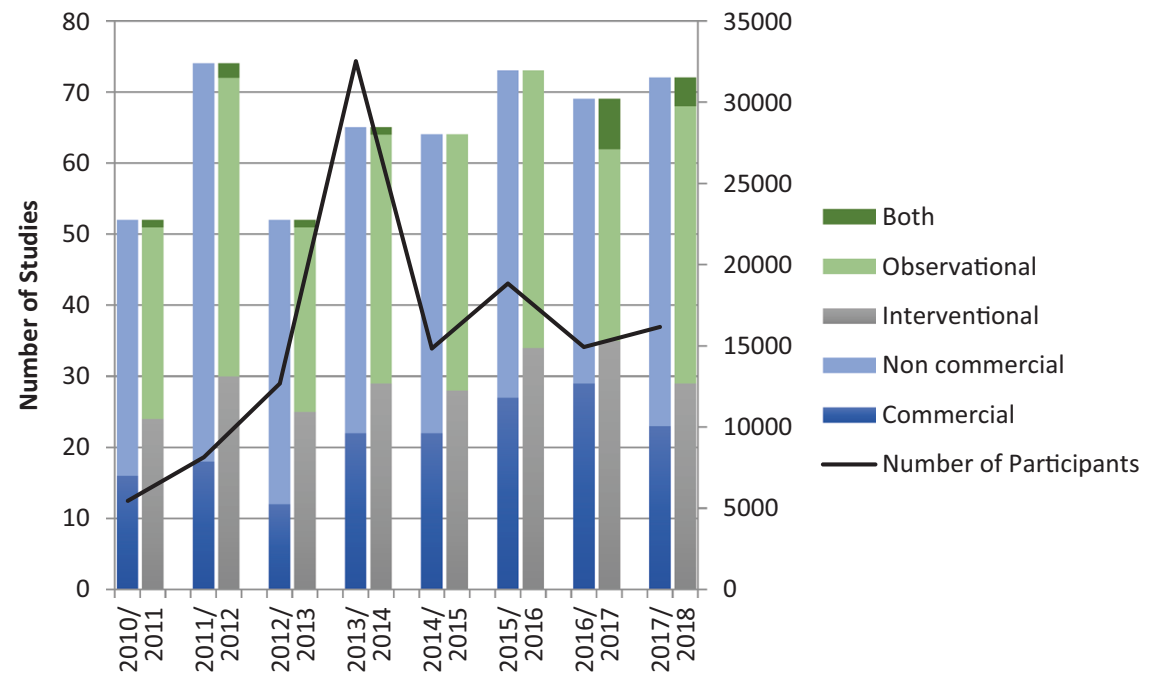

\section{Methods}

Supported by the NIHR's Business Intelligence Unit (KB), the database of the UK CRN portfolio was accessed and Ophthalmology studies that had been open to recruitment at any time between 1 April 2010 and 31 March 2018 were selected [4]. Studies that had closed to recruitment prior to 1 April 2010 were not included as in the early phase of creation of the UK CRN portfolio, details of commercial studies in particular were incompletely captured. As Ophthalmology encompasses a wide range of topics each study was classified by sub-specialty by two researchers (SD and EL) with specialist knowledge of vision science. The study protocol was scrutinised and in the event of discordance in assigning the sub-specialty classification, a third researcher (RB) arbitrated. Studies were also categorised according to participant age (adults or paediatric or both), and by the gender of the Chief Investigator of the studies. Finally, studies were grouped into those that involved genetic investigations and those that did not. Studies on the portfolio were also classified as commercial or non-commercial and by design into interventional or observational studies. Frequency distribution charts were prepared.

\section{Results}

Over 7 years between 1 April 2010 and 31 March 2018, 137,377 participants were recruited into Ophthalmology portfolio studies across the UK, with an increase in numbers recruited from 5485 participants in 2010/2011 to 16,182 in $2017 / 18$ (Fig. 2). An average of 15,457 participants were recruited per year (range: 5485-32,573). Recruitment in $2013 / 2014$ was particularly high at 32,573 participants on account of a single cohort commercial study into which 19,100 people were recruited. Fifty-two studies were entered onto the portfolio in 2010/2011, increasing to 72 in $2017 / 18$, the majority of studies were non-commercial with a non-commercial:commercial ratio that ranged between 3.3:1 in 2012/2013 and 1.4:1 in 2016/17. The contribution of commercial studies to the total number of studies per year increased during this time period. More studies were observational in design than interventional with an observational:interventional ratio that ranged between 1.4:1 and $0.7: 1$ for these year points.

In 2017/2018, 76\% (86 of 113) of hospitals with acute eye care services in England were recruiting into at least one NIHR portfolio Ophthalmology study. This figure had increased from 2016/2017 (69\%) and 2015/16 (57\%). The number of participants recruited in each of the 15 English LCRNs over the 8-year period ranged from 1838 to 36,266. The English LCRN figures are presented alongside recruitment among the devolved nations of the UK in Fig. 3. The highest-recruiting region was North-West Coast, recruiting 36,266 participants (one particular study recruited 19100 participants [study ID 14595]), followed by 24,509 participants recruited in the North Thames region, the location of the only Biomedical Research Centre dedicated solely to Ophthalmology, the NIHR Moorfields Biomedical Research Centre.

Eighty studies $(13.8 \%)$ had a genetic component. The majority of studies $(81.7 \%)$ included adults only. Forty-two studies included children and 64 included both adults and children.

Examining the sub-specialty distribution within Ophthalmology (Fig. 4), the majority of studies (41\%) enrolled patients with diseases of the retina, followed by glaucoma $(17 \%)$, anterior segment and cataract (13\%), and ocular inflammation $(6 \%)$. Of the retinal studies, the principal 
Fig. 3 Numbers of participants (and number recruited per 100,000 population) recruited into UK CRN Ophthalmology portfolio studies in each of the 15 Local Clinical Research Networks and the devolved nations between 1 April 2010 and 31 March 2018
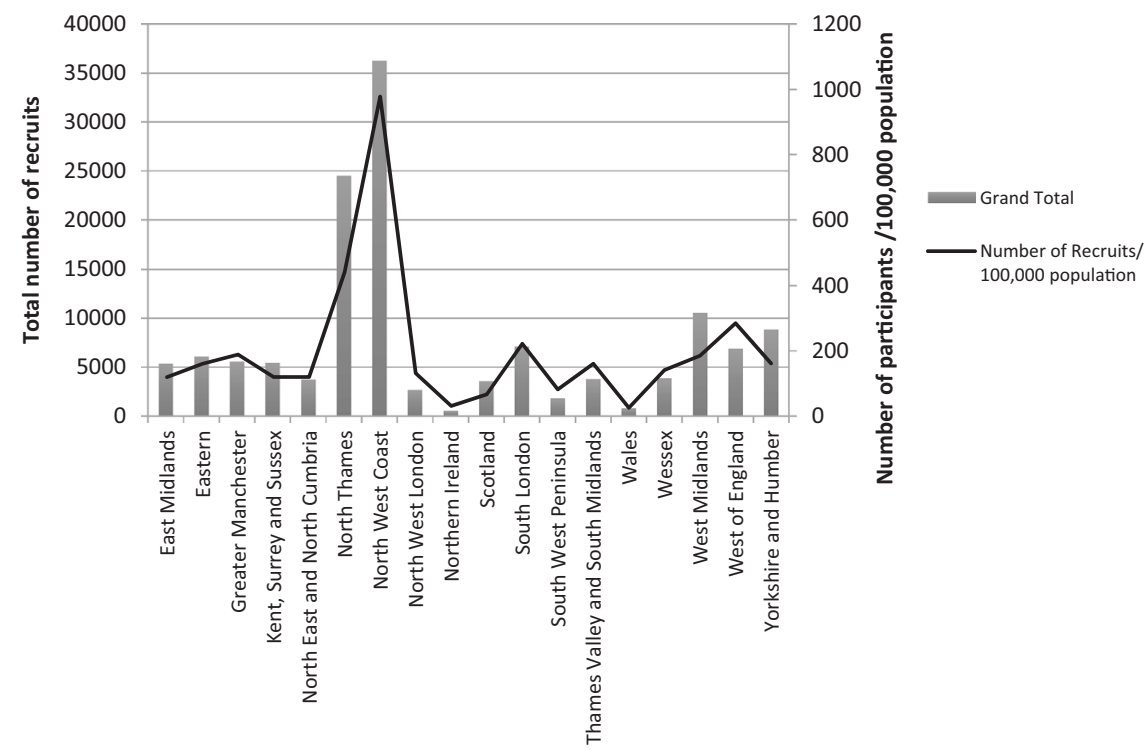

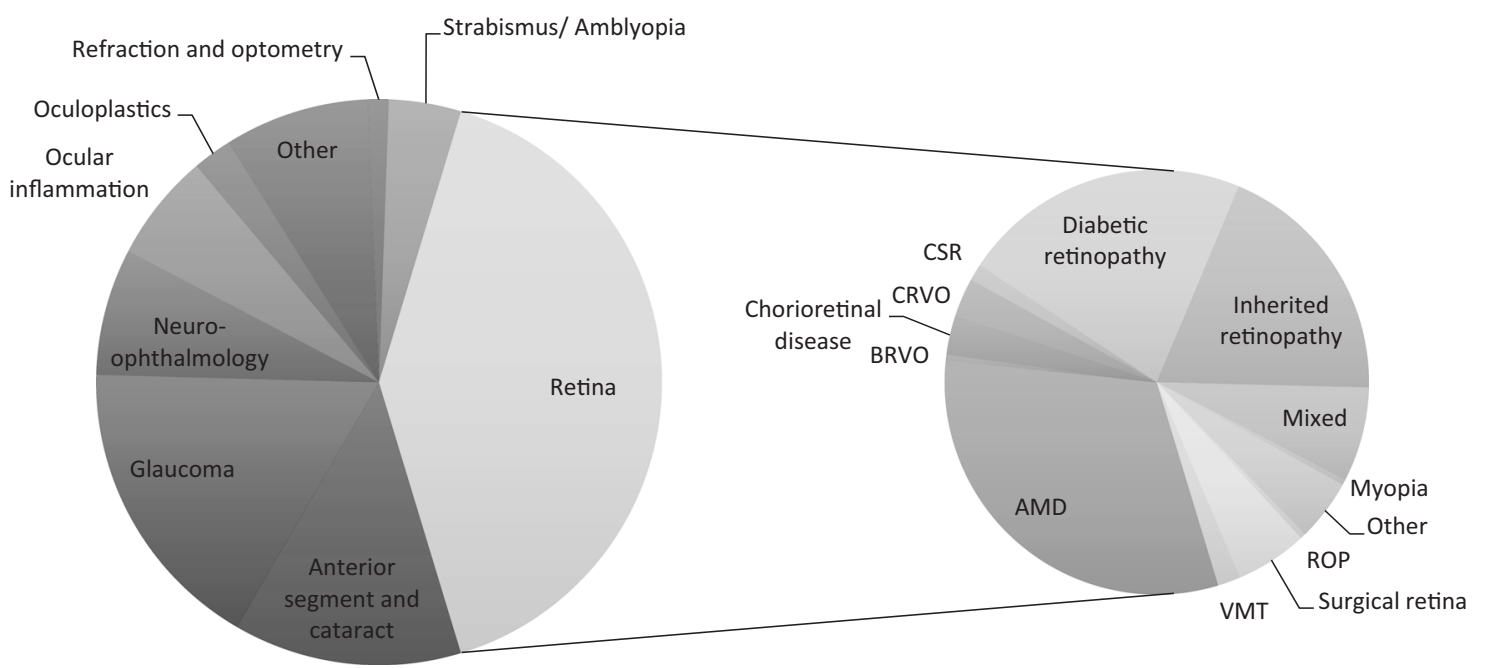

Fig. 4 Numbers of UK CRN Ophthalmology portfolio studies classified by sub-specialty that were open between April 2010 and 31 March 2018

disease groups were age-related macular degeneration (13\% of all studies), diabetic retinopathy (9\%) and inherited retinopathy (6\%). Studies of thyroid eye disease were included in the 'Other' category.

By sub-specialty, there were differences in temporal trends in terms of both absolute numbers and proportion of studies from the different sub-specialties over the period of enquiry (Appendix 2 Supplementary materials). The number and proportion of studies of diseases of the retina rose over time. Although the numbers of studies were lower in the other sub-specialties, there was a steady increase in the anterior segment and cataract group. Studies of neuroophthalmology and glaucoma remained relatively static, while those dealing with ocular inflammation studies declined slightly.
Over the 8-year time period, there were differences between sub-specialties in terms of the non-commercial: commercial study ratio. For example, ocular inflammation studies had more commercial involvement (0.9:1), compared with the other sub-specialties such as glaucoma (2.3:1), retina (1.7:1), and anterior segment and cataract (2.4:1). There were no commercial refraction and optometry studies. There was a gradual increase in the proportion of retina studies that are commercial compared to non-commercial between 2010/11 (6 studies: 9 studies) and 2016/17 (16:14), falling marginally in 2017/2018 (10:14).

Funders of studies in the portfolio are presented in Fig. 5. Among non-commercial studies (Fig. 5a), fight for sight (FFS) was responsible for funding $\sim 15-30 \%$ of studies in each of the years examined, with NIHR funding 
Fig. 5 Sources of funding of UK CRN Ophthalmology portfolio studies by year between April 2010 and 31 March 2018. a Sources of funding of UK CRN Ophthalmology portfolio studies by year between April 2010 and 31 March 2018: non-commercial studies. b Sources of funding of UK CRN Ophthalmology portfolio studies by year between April 2010 and 31 March 2018: commercial studies a

Top Funders of Commercial Studies Opening Per Year

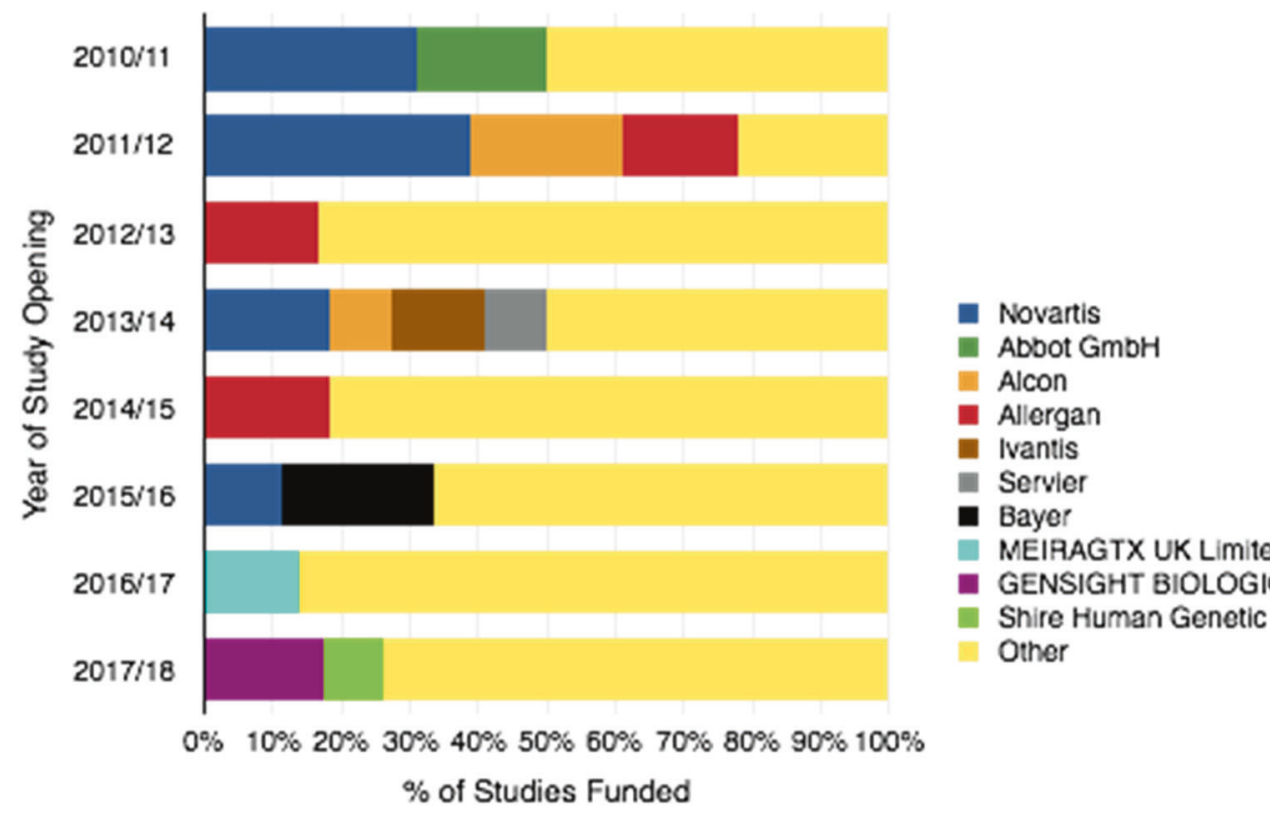

b Top Funders of Non-Commercial Studies Opening Per Year

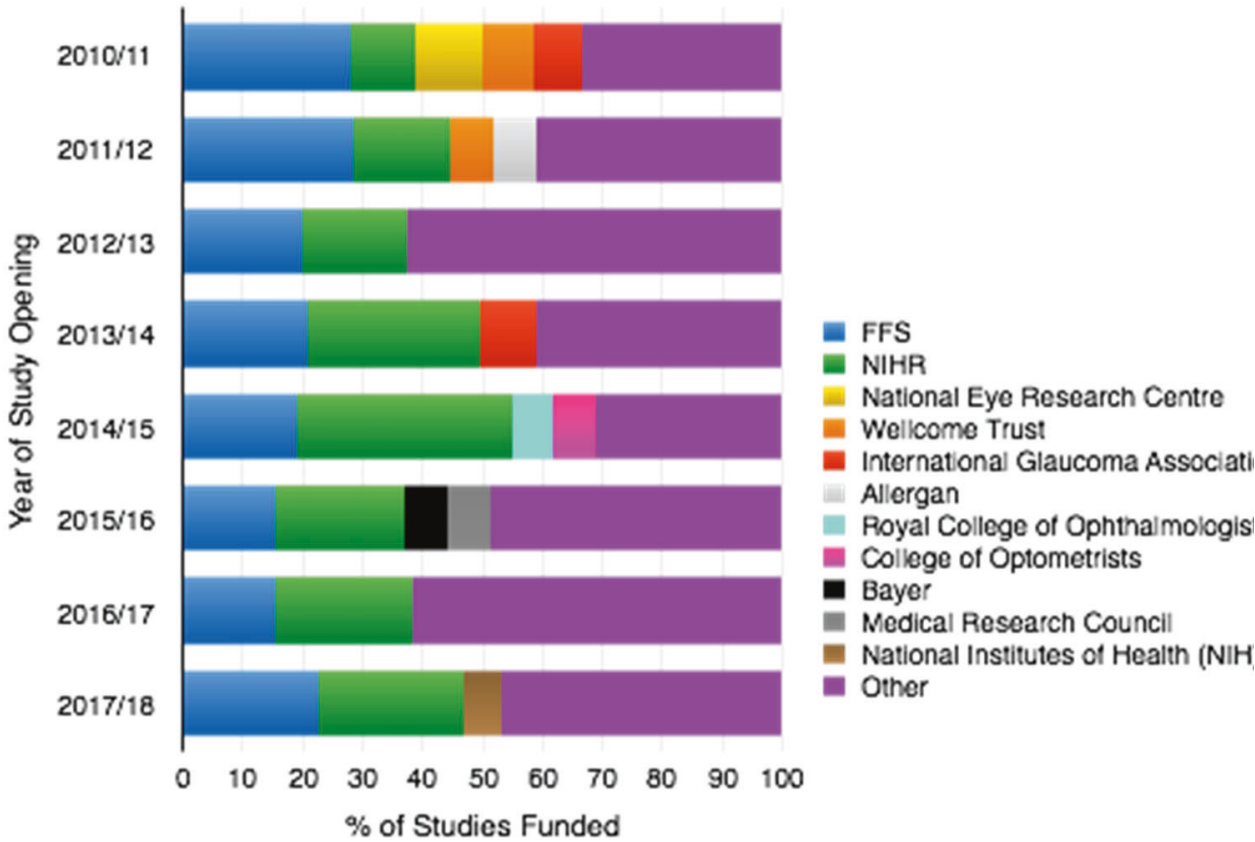

a further 10-35\%. Funders of commercial studies are given in Fig. $5 b$.

Across the 8-year period from $2010 / 11$ to $2017 / 18$, $68 \%$ of non-commercial studies recruited within the anticipated time set by the study and also recruited to or exceeded the target number of participants set by the study. For commercial studies, this figure was 55\%. This overall figure disguises the improvement in achieving recruitment to time and target over this period, which is presented in Appendix 3 Supplementary materials. For example, in the past 6 years, the recruitment to time and target for non-commercial and commercial studies has been $71 \%$ and $58 \%$, respectively. A proportion of those studies that missed both time and recruitment targets were 'near-misses', where studies achieved $90-100 \%$ in respect either of time or target or both. A total of $21 \%$ non-commercial studies and $27 \%$ 
of commercial studies that missed $100 \%$ target were 'nearmisses', over the 8-year period.

The gender of the Chief Investigator was known for 379 studies. The trend across the years for proportion of male or female chief investigators are shown in Appendix 4 Supplementary materials. Overall, there were more male chief investigators for every year analysed. For example, a third of studies in 2017/18 were led by a female chief investigator. There was no clear trend over the study period in this gender imbalance.

\section{Discussion}

Clinical research in the UK is essential for improving care for patients, for example, by providing evidence on the efficacy of new healthcare treatments. The NIHR aims to increase the opportunities for patients and the public to participate in, and benefit from, research. Ophthalmology is clearly a very active research area within the UK CRN portfolio, recruiting an average of 15,000 patients into clinical trials within the NHS per year, with consistent growth over the past 7 years. The majority of hospitals (76\%) that deliver acute eye care services are participating in recruitment of patients to UK CRN Ophthalmology studies yet there are hospitals which are still not involved. Increasing hospital participation has been a focus ('Specialty Objective') of the NIHR Ophthalmology NSG in recent years. Ophthalmology remains the single busiest out-patient specialty in hospitals in the UK (for example, there are a million out-patient visits for glaucoma per year [5]). It is therefore important that patients have access to the benefits of clinical research that include new medications, devices, and diagnostic technology. Ophthalmology departments also benefit from participation in UK CRN portfolio studies with funding allocation available for staff and training. With the advent of online clinical trial search systems (e.g. UK Clinical Trials Gateway) [6], there has been a growing awareness among patient groups of potential ocular research projects, and a drive from patients and their carers to seek out trials relevant to their ocular disease. Recent reports involving bowel cancer survival [7] have demonstrated that hospitals that engage in clinical research have better outcomes. This may well also be the case for Ophthalmology and underscores the need to encourage involvement of the remaining 24\% (2017/18 data) of UK Ophthalmology Departments who are not currently participating in UK CRN Ophthalmology Portfolio studies. The work of the NIHR Ophthalmology NSG in encouraging more involvement by the Royal College of Ophthalmologists in integrating research into clinical care through a number of initiatives that include regular symposia at College congresses, web-based resources for all clinicians, and awards to highly active and inspirational clinical researchers and trainees, has undoubtedly contributed to awareness of the Ophthalmology portfolio and its growth. This has also been assisted by regular review of the portfolio of commercial and non-commercial studies with the NIHR Portfolio Management team (EC) to maximise recruitment to open studies and to detect studies in difficulty early and target support (https://www.rcophth.ac.uk/professional-resources/ research-hub/the-nihr-ophthalmology-clinical-researchnetwork/).

Financial benefits of participation in NHS research are substantial. A report by KPMG estimated that for commercial studies, NHS Trusts receive an average of $£ 6658$ per patient in revenue from sponsor companies, and a pharmaceutical cost saving of $£ 5250$ per patient recruited to each clinical study [8]. This equated to the estimated totals of $£ 176$ million of commercial income and $£ 16$ million of pharmaceutical cost savings across the commercial CRN Portfolio in 2014/15. Our analysis has demonstrated the importance of commercial involvement in the funding of Ophthalmology clinical studies. The proportion of studies that are commercial was small in the early years of the Ophthalmology NSG, yet a drive to increase means this proportion has risen to a third of the Ophthalmology study portfolio. Another aim of the NIHR is to maximise the research potential of the NHS to contribute to the economic growth of the country through the life science industry [9]. We have seen a gradual increase in the proportion of commercial studies, particularly in the category of retinal disease, when compared to numbers of non-commercial studies over this time period. Ocular inflammation studies also have a significant commercial involvement. However, this analysis has shown that in some sub-specialty areas such as glaucoma and anterior segment and cataract, commercial investment in UK CRN portfolio studies is much less marked. Given the contribution of these two disease groups, respectively, to vision impairment [10] and ocular morbidity [11], and the substantial commercial interests in these diseases, further commercially funded research development in these areas would be welcome. Ophthalmology remains a leader among other specialties within the UK in terms of research into novel research areas such as gene therapies [12], novel drug delivery systems [13], robotic surgery [14] and artificial intelligence [15], all of which have enormous growth potential.

Non-commercial funding for Ophthalmology research is a major challenge within the UK. In a report published by The Macular Society, of a total of $£ 2025.2$ million spent by charities, research councils and government on health research, only £22.7 million was spent on eye research in 2014. Despite there being considerable turnover by sight loss charities per year ( $£ 774.5$ million income in 2014), there was a relatively small financial contribution to UK 
research within this sector, the largest donor being Fight for Sight, which contributed $£ 3.6$ million of a total of $£ 9.6$ million spent by sight loss charities on UK medical research in 2014 [16]. Closer collaboration between patient groups, eyecare charities and the research community to build on prior work to identify key research priorities is underway; an excellent example of this is the James Lind Alliance where stakeholders (patients, carers, third sector, Ophthalmologists, Optometrists) came together to categorise research priorities in a series of meetings. Innovative partnerships to boost research funding through commercial, philanthropic and charitable sources need to be explored, in order for research into eye disease to match the success of other UK-based research pipelines as seen in cancer and diabetes research. Sensory organ diseases rank seventh in the global ranking of DALYs by cause [17]. We should be aiming to ensure that research funding matches this high health burden ranking. Cross-cutting research that considers the impact of eye disease and vision impairment on related disease areas, for example, cognitive decline and frailty, and comorbidities may augment the importance of eye research in the eyes of potential funders.

A recent focus of the NIHR has been to assess whether research is active in areas of the greatest need. In our analysis of numbers of Ophthalmology study recruits/100,000 population (Fig. 3), it is clear that there is wide geographical variation in research participation even when discounting North Thames (given the presence of a Biomedical Research Centre devoted to vision science in that location). With the development of networks of Ophthalmology electronic record systems such as the National Ophthalmology Database [18], UK Ophthalmology has the potential to better understand its areas of met need. However, without accurate population-based data on distribution of eye disease and its risk factors, we are unable to map research activity to areas of greatest need. This is the rationale for the UK National Eye Health Survey that is currently in preparation.

NIHR CRN and its Ophthalmology NSG has shown itself capable of delivering very large multicentre studies across the UK that benefit from NIHR support costs which have had very significant national and international impact on patient outcomes [19-25]. This demonstrates that UK centres work together cohesively to produce high-quality research. The NIHR has contributed to the development and strengthening of these networks, providing local and national guidance, as well as fostering and encouraging close working partnerships with the third sector and commercial entities. These studies require very substantial organisation of the infrastructure at each of these hospital sites for the duration of each study. Retention of this infrastructure to rapidly deliver further studies at these sites is a major focus of the NIHR Ophthalmology NSG drawing on experiences of other networks in other countries, which have pipelines of research protocol development, funding streams and research delivery [26]. Ten years ago, studies were scrutinised by members of the NIHR Ophthalmology NSG prior to inclusion onto the portfolio for quality, presence of a clear research question and relevance to the NHS and to ensure that funding was in place. In 2014, this process was streamlined to only involve studies that were not funded by automatically eligible funding streams. From the point of securing funding and making the application for inclusion, the time taken to recruit has also reduced with improvements to the governance processes (this was also attributable to the work of the NIHR CRN teams) who developed the pathways and standard procedures to facilitate streamlined progression through ethics and NHS Research and Development hospital trust approvals. The targeting of NIHR CRN resources to non-research active centres allowed these NHS units to participate. Strategic support to specific clinical sites (often guided by the NIHR Ophthalmology NSG during the recruitment phase of failing studies) has helped markedly turn round many of these studies. The recruitment to time and target presented in Appendix 3 (Supplementary Materials) demonstrates this improvement within Ophthalmology studies over the last 8 years yet there is still room for improvement, particularly with commercial studies.

The high participation rate of Ophthalmology departments (79\% in 2017/18) in hospitals across the UK demonstrates the willingness of ophthalmologists, optometrists, ophthalmic technicians and nurses to get involved in these interesting and innovative studies. The Health and Social Care Act of 2012 placed a statutory duty to promote research in the NHS and the Ophthalmology community have demonstrated how clinical research can be successfully embedded into clinical care. The Royal College of Physicians' 'Research for All' report identified areas which could be improved in order to maximise participation [27]. One of these areas may involve exploring the gender differences in research activity, a part of which was reflected in our finding that there were fewer female Chief Investigators among NIHR Ophthalmology portfolio studies than men. This ratio for the years 2015/16 (3.5:1) and 2016/17 (1.5:1) is not that different to the ratio of male:female ophthalmology consultants in the UK in 2016, which has been reported as 2.8:1 [28]. Several reports have identified underrepresentation of women in medical research [29]. Another area of development sponsored by the NIHR Ophthalmology NSG has been the development of Ophthalmology Trainee Research Networks which have successfully developed large multicentre studies and are continuing to expand across the UK [30]. Direct experience of clinical 
studies by trainees, often working as principal investigators, prepares them early in their careers to integrate research into clinical care.

In conclusion, this analysis has demonstrated high levels of clinical research activity in the field of Ophthalmology and its sub-specialties over the past 8 years, with enthusiastic participation by hospital departments, their staff and their patients across the UK. The study highlights some subspecialties of Ophthalmology that carry substantial morbidity and a very high burden on NHS services, which do not currently attract the clinical research studies and commercial interest that they deserve. More patient-centred research into these areas and a more coordinated approach to matching research activity to need are areas of ongoing development. However, it is important to note that the first key steps in achieving the goal of embedding research into every day clinical care have been enabled by NIHR and that the Ophthalmic specialist network appears to work well with increases of participant recruitment year on year across all four nations of the UK.

\section{Summary}

\section{What was known before}

- The National Institute for Health Research (NIHR) has transformed research in the United Kingdom's National Health Service (NHS) since its establishment in 2006.

- The NIHR CRN Ophthalmology National Specialty Group (NSG) oversees, monitors and supports the delivery of Ophthalmology portfolio studies on the UK CRN portfolio.

\section{What this study adds}

- Reports on the composition and performance of the portfolio of Ophthalmology research studies in the UK CRN over the past 8 years.

- Identifies an increasing proportion of commercial study activity in recent years.

- Identifies certain sub-specialty areas of Ophthalmology that cause significant health burden but which are currently underserved by patient-centred research.

- Highlights the high participation of the country's hospitals in Ophthalmology research studies and an average of 15,500 patients recruited into studies every year with ongoing improvements in performance.

\section{Compliance with ethical standards}

Conflict of interest The authors declare that they have no conflict of interest.
Members of the NIHR Ophthalmology Specialty Group: Jane Ashworth, Clare Bailey, Susan Downes, Louise Downey, Sheena George, Irene Gottlob, Christopher Hammond, Simon Harding, Jonathan Jackson, Andrew Lotery, Martin McKibbin, Luke Membrey, Pádraig J. Mulholland, Nishal Patel, George M. Saleh, Roshini Sanders, Giuliana Silvestri, Velota Sung, Deepali Varma, Marcella Votruba, Emma Worrell, Brinda Shah, Ian Nickson, Michael Beresford, Vanessa Poustie, Emma Chambers, Sarah Cooper, Cathy Yelf, Geraldine Hoad, Michele Acton, Louise Gow, Matt Broom, Christine Dickinson, Padraig Mulholland, Manjo Doug, Robert Barry

\section{References}

1. http://www.legislation.gov.uk/ukpga/2012/7/pdfs/ukpga_ 20120007_en.pdf. Accessed 1 July 2018.

2. http://www.crncc.nihr.ac.uk/about_us/processes/portfolio. Accessed 1 July 2018.

3. Bourne R, Gale R. The Ophthalmology Specialty Group and the National Institute for Health Research: the first 6 years. Eye. 2013;27:457-60.

4. NIHR Clinical Research Network Open Data Platform. www.odp. nihr.ac.uk. Accessed 1 July 2018.

5. Azuara-Blanco A, Burr J, Thomas R, Maclennan G, McPherson S. The accuracy of accredited glaucoma optometrists in the diagnosis and treatment recommendation for glaucoma. Br J Ophthalmol. 2017;91:1639-43

6. https://ukctg.nihr.ac.uk. Accessed 1 July 2018.

7. Downing A, Morris EJ, Corrigan N, Sebag-Montefiore D, Finan PJ, Thomas JD, et al. High hospital research participation and improved colorectal cancer survival outcomes: a population-based study. Gut. 2017;66:89-96.

8. https://www.nihr.ac.uk/life-sciences-industry/documents/NIHR\% 20CRN\%20Impact\%20and\%20Value\%20FINAL\%20REPORT vSTC_160908_FOR\%20EXTERNAL\%20USE.pdf. Accessed 1 July 2018.

9. Bell J. Life sciences: industrial strategy. https://www.gov.uk/ government/publications/life-sciences-industrial-strategy. Accessed 1 July 2018.

10. Bourne RRA, Jonas JB, Bron AM, Cicinelli MV, Das A, Flaxman $\mathrm{SR}$, et al. Prevalence and causes of vision loss in high-income countries and in Eastern and Central Europe in 2015: magnitude, temporal trends and projections. Br J Ophthalmol. 2018;102: 575-85.

11. Stapleton F, Alves M, Bunya VY, Jalbert I, Lekhanont K, Malet F, et al. TFOS DEWS II epidemiology report. Ocul Surf. 2017;15:334-65.

12. Xue K, Jolly JK, Barnard AR, Rudenko A, Salvetti AP, Patrício MI, et al. Beneficial effects on vision in patients undergoing retinal gene therapy for choroideremia. Nat Med. 2018;24:1507-12.

13. A comparison of Bimatoprost SR to Selective Laser Trabeculoplasty in patients with open-angle glaucoma or ocular hypertension. https://clinicaltrials.gov/ct2/show/NCT02507687?term= bimatoprost\&cond $=$ Glaucoma $\% 2 \mathrm{C}+$ Open-Angle\&rank $=1$. Accessed 1 July 2018.

14. Edwards TL, Xue K, Meenink HCM, Beelen MJ, Naus GJL, Simunovic MP, et al. First-in-human study of the safety and viability of intraocular robotic surgery. Nat Biomed Eng. 2018;2: 649-56.

15. De Fauw J, Ledsam J, Romera-Paredes B, Nikolov S, Tomasev N, Blackwell S, et al. Clinically applicable deep learning for diagnosis and referral in retinal disease. Nat Med. 2018;24: $1342-50$

16. The Macula Society. Age-related macular degeneration: collaborating to find a cure. Appendix A. Charity, Research Council and Government funding by Health Category. UK Health 
Research Analysis 2014. https://www.macularsociety.org/colla borating-find-cure. Accessed 1 July 2018.

17. GBD 2016 DALYs and HALE Collaborators. Global, regional, and national disability-adjusted life-years (DALYs) for 333 diseases and injuries and healthy life expectancy (HALE) for 195 countries and territories, 1990-2016: a systematic analysis for the Global Burden of Disease Study 2016. Lancet. 2017;390: $1260-1344$

18. National Ophthalmology Database Audit. https://www.nodaudit. org.uk. Accessed 1 July 2018.

19. Sivaprasad S, Prevost AT, Vasconcelos JC, Riddell A, Murphy C, Kelly $\mathrm{J}$, et al. Clinical efficacy of intravitreal aflibercept versus panretinal photocoagulation for best corrected visual acuity in patients with proliferative diabetic retinopathy at 52 weeks (CLARITY): a multicentre, single-blinded, randomised, controlled, phase 2b, non-inferiority trial. Lancet. 2017;389:2193-203.

20. Azuara-Blanco A, Banister K, Boachie C, McMeekin P, Gray J, Burr J, et al. Automated imaging technologies for the diagnosis of glaucoma: a comparative diagnostic study for the evaluation of the diagnostic accuracy, performance as triage tests and cost-effectiveness (GATE study). Health Technol Assess. 2016;20:1-168.

21. Garway-Heath DF, Crabb DP, Bunce C, Lascaratos G, Amalfitano $\mathrm{F}$, Anand $\mathrm{N}$, et al. Latanoprost for open-angle glaucoma (UKGTS): a randomised, multicentre, placebo-controlled trial. Lancet 2015;385:1295-304.

22. Sivaprasad S, Vasconcelos JC, Prevost AT, Holmes H, Hykin P, George S, et al. Clinical efficacy and safety of a light mask for prevention of dark adaptation in treating and preventing progression of early diabetic macular oedema at 24 months (CLEOPATRA): a multicentre, phase 3, randomised controlled trial. Lancet Diabetes Endocrinol. 2018;6:382-91.

23. Protocol 14PRT/06545: LEAVO: a multicentre phase III double-masked randomised controlled non-inferiority trial comparing the clinical and cost effectiveness of intravitreal therapy with ranibizumab (Lucentis) vs aflibercept (Eylea) vs bevacizumab (Avastin) for macular oedema (MO) due to central retinal vein occlusion (CRVO). LEAVO Trial (2016) Lancet http://www.thelancet.com/protocol-reviews/ 14PRT-06545. Accessed 1 July 2018.

24. Azuara-Blanco A, Burr J, Ramsay C, Cooper D, Foster PJ, Friedman DS, et al. Effectiveness of early lens extraction for the treatment of primary angle-closure glaucoma (EAGLE): a randomised controlled trial. Lancet. 2016;388:1389-97.

25. Chakravarthy U, Harding SP, Rogers CA, Downes SM, Lotery AJ, Culliford LA, et al. Alternative treatments to inhibit VEGF in age-related choroidal neovascularisation: two-year findings of the IVAN randomised controlled trial. Lancet. 2013; 382:1258-67.

26. Diabetic Retinopathy Clinical Research Network. http://drcrnet.ja eb.org. Accessed 1 July 2018.

27. Royal College of Physicians. Research for all. https://www. rcplondon.ac.uk/projects/outputs/research-all. Accessed 1 July 2018.

28. The Royal College of Ophthalmologists. Workforce census 2016: a picture of the size and shape of the UK ophthalmic medical workforce. https://www.rcophth.ac.uk/2017/03/workforce-census2016-a-picture-of-the-size-and-shape-of-the-uk-ophthalmicmedical-workforce/. Accessed 1 July 2018.

29. Ovseiko PV, Greenhalgh T, Adam P, Grant J, Hinrichs-Krapels $\mathrm{S}$, Graham KE, et al. A global call for action to include gender in research impact assessment. Health Res Policy Syst. 2016;14:50

30. The Royal College of Ophthalmologists. NIHR Information for Ophthalmology Trainees. https://www.rcophth.ac.uk/professionalresources/research-hub/the-nihr-ophthalmology-clinical-researchnetwork/useful-information-for-eye-care-professionals/nihrinformation-for-ophthalmology-trainees/. Accessed 1 July 2018. 